Research Paper

\title{
ANG Promotes Proliferation and Invasion of the Cell of Lung Squamous Carcinoma by Directly Up-Regulating HMGA2
}

\author{
Li Xu*, Wei-Lin Liao*, Qi-Jue Lu*, Chun-Guang Li, Yang Yuan, Zhi-Yun Xu, Sheng-Dong Huang, He-Zhong \\ Chen $\bowtie$ \\ Department of Cardiothoracic surgery, Changhai Hospital, Second Military Medical University, Shanghai, People's Republic of China. \\ * These authors contributed equally to this work. \\ $\bowtie$ Corresponding author: He-Zhong Chen, drchenhz@163.com, 168 Changhai Road, Shanghai, P.R. China, 200438.
}

() Ivyspring International Publisher. Reproduction is permitted for personal, noncommercial use, provided that the article is in whole, unmodified, and properly cited. See http://ivyspring.com/terms for terms and conditions.

Received: 2015.11.16; Accepted: 2016.03.15; Published: 2016.04.28

\begin{abstract}
Objective: To determine the mechanism of Angiogenin(ANG) function involved in the carcinogenesis of lung squamous cell carcinoma.

Methods: 12 patients' normal tissue and cancerous tissue were collected. ANG expression in the squamous cell carcinoma of the lung was evaluated by $\mathrm{QRT}-\mathrm{PCR}$ and western-blot. The regulation of ANG on proliferation, migration, invasion, and apoptosis of SK-MES-1 cells were analyzed by Cell Counting Kit-8, Transwell migration chamber, Transwell invasion chamber, and Annexin V-FITC assay, respectively. PCR array was utilized for screening potential target genes of ANG. Chromatin immunoprecipitation(ChIP) assays and luciferase assay were adopted for investigation of ANG's direct regulation on HMGA2.

Results: ANG expression is increased in the squamous cell carcinoma of the lung tissue. In vitro experiments results indicated that overexpression of ANG promotes proliferation and invasion capability of SK-MES-1 cells. The candidate proliferation, migration, and invasion related ANG target gene found was HMGA2, expression levels of which were also enhanced in lung squamous cell carcinoma tissue. The direct regulation of ANG on HMGA2 was verified by ChIP and luciferase assay results. Furthermore, down-regulating HMGA2 significantly alleviated the suppression effects of ANG on proliferation, migration, and invasion of SK-MES-1 cells.

Conclusions: Our data illustrated the mechanisms that ANG promoted the cell of SQCLC proliferation, migration, and invasion capacity via directly up-regulating HMGA2.
\end{abstract}

Key words: Angiogenin, lung squamous cell carcinoma, HMGA2.

\section{Introduction}

The lung cancer, as the most common tumor among all the malignant tumors, causes the most cancer deaths worldwide[1]. While it is the fact that the prognosis of lung cancer is poor, despite significant therapeutic improvements have been made in recent years. However, it is studied that preventing the invasion and metastasis of cancerous cells plays an important role in lowering the morbidity and mortality of this disease. Squamous cell carcinoma of the lung currently has ranked as the second most frequent histological subtype of non-small cell lung cancer and accounted for about 40 $\%$ of all lung cancers[2] [3].

Angiogenin (ANG), as a 14, 400 Da angiogenic ribonuclease of the RNase A superfamily, is up-regulated in a variety of human cancers, which has a 33\% amino acid identity and an overall homology of $56 \%$ to that of RNase-A[4]. Angiogenin (ANG) has been shown to play a permissive role in tumor angiogenesis[5]. Recent research indicates that ANG also directly stimulates cancer cell proliferation by enhancing rRNA transcription[6-9], thus has been 
linked to worse clinical prognosis in one study[10]. ANG cleaves tRNA and promotes ribosomal RNA transcription affecting a wide variety of responses including proliferation, cell invasion and invasion and tube formation in the nucleus, and nuclear ANG cannot be detected in normal, non-endothelial cells[8]. However, few consolidated report has been conducted to investigate the concrete mechanism and the pathways downstream of ANG. High mobility group AT-hook 2 (HMGA2) is involved in various biological processes such as the formation of the embryo and the development of malignant cancer[11]. Members of the HMGA protein family are often referred to as "architectural transcription factors" [12], because of their ability to regulate expression of a large number of target genes through alteration of chromatin structure[13]. HMGA2 gene has shown to be of prognostic relevance in several other cancer entities[14-16].

Based on this knowledge, the current study examines the impact of ANG-HMGA2 pathway on the proliferation and invasion of the squamous cell carcinoma of the lung cell line SK-MES-1 and discusses its possible mechanisms of action, as clarified that our results provide experimental evidence for the inclusion of ANG-based targeted therapies in the clinical treatment of SQCLC.

\section{Material and Methods}

\section{Tissue collection}

The cancerous and benign specimens, frozen fresh for western blot analysis and qRT-PCR, of SQCLC were obtained from 12 patients who underwent lung cancer resection procedures at Changhai Hospital in 2014. All cancerous and matching non-cancerous samples used for this study were provided by the Clinical and Experimental Pathology of the Research Centre of Changhai Hospital, Shanghai, China where initial H\&E staining and histologic diagnoses were performed after standard surgical primary tumor resection. This study was conducted in accordance with the Declaration of Helsinki and was approved by the Medical Ethics Committee of Changhai Hospital, with all patients written informed consent.

\section{Cell culture}

SK-MES-1 cells were obtained from the Cancer Cell Repository (Shanghai Cell Bank, Shanghai, China), which were maintained in DMEM supplemented with $10 \%$ (v/v) heat-inactivated fetal bovine serum (Gibco-BRL) and antibiotics $(100 \mathrm{U} / \mathrm{ml}$ penicillin and $100 \mathrm{U} / \mathrm{ml}$ streptomycin; Hyclone Laboratories, Inc., Logan, UT, USA) at $37^{\circ} \mathrm{C}$ in a humidified atmosphere of $5 \% \mathrm{CO} 2$.

\section{RNA Interference and ANG overexpression}

The ANG-specific short hairpin RNA adenovirus vector (shANG), the HMGA2-specific short hairpin RNA adenovirus vector (shHMGA2) and the negative control short hairpin RNA adenovirus vector (shScramble) were purchased from Genechem Co., Ltd (Shanghai, China). The sequences of shANG were: sense strand1: 5'-CCGCGGGATGACAGATAC TGTGAAGAGATTCACAGTATCTGTCATCCCG-3' and antisense strand1: 5'-AACGGGATGACAGATAC TGTGAATCTCTTCACAGTATCTGTCATCCCGC-3'; sense strand2: 5'-CCGGATCCCAGGCTCGTTCTTTG GAGACAAAGAACGAGCCTGGGATCC-3' and antisense strand2: 5'-AAGGATCCCAGGCTCGTTCT TTGTCTCCAAAGAACGAGCCTGGGATCC-3'. The sequences of shHMGA2 were: sense strand1: 5'-CACCGCCACAACAAGTTGTTCAGAACGAATT CTGAACAACTTGTTGTGGC-3' and antisense strand1: 5' - AAAAGCCACAACAAGTTGTTCAGAA TTCGTTCTGAACAACTTGTTGTGGC -3'; sense strand2: 5'-TAGGAGGAAACTGAAGAGACATCTC GAGATGTCTCTTCAGTTTCCTCCTTTTTTC-3' and antisense strand2: 5'-TCGAGAAAAAAGGAGGAAA CTGAAGAGACATCTCGAGATGTCTCTTCAGTTT

CCTCCTA-3'. An adenovirus vector co-expressing ANG and green fluorescent protein (Ad-ANG) and a control adenovirus vector expressing green fluorescent protein (Ad-GFP) were constructed using AdEasy system (Genechem Co., Ltd Shanghai, China).

Quantitative Real-Time Reverse-Transcription Polymerase Chain Reaction (qRT-PCR)

Total RNA was extracted and cDNA was synthesized using the PrimeScript RT Reagent Kit (TaKaRa Bio). SYBR Premix EX Taq (TaKaRa Bio) was used for quantitative real-time polymerase chain reaction performed using a LightCycler ${ }^{\circledR} 480$ Real-Time PCR System (Roche Diagnostics). The primers for each gene were as follows: 18s rRNA forward: 5'-CGGACACGGACAGGATTGAC-3'; 18s rRNA reverse: 5'-GCATGCCAGAGTCTCGTTCG-3'; ANG forward: 5'-GGACTTGTTCTGAGGCCGAG-3'; ANG reverse: 5'-CCAGCACGAAGACCAACAAC-3'; HMGA2 forward: $5^{\prime}$-AGCAGCAGCAAGAACCAAC C-3'; HMGA2 reverse: 5'-CCTGAGCAGGCTTCTTCT GA-3'. The amplification conditions were as follows: 1 cycle of $95^{\circ} \mathrm{C}$ for 30 seconds and 40 cycles of $95^{\circ} \mathrm{C}$ for 5 seconds followed by $60^{\circ} \mathrm{C}$ for 30 seconds. The expression levels of the target genes relative to the control were determined by the $2^{-\Delta \Delta \mathrm{CT}}$ method. $18 \mathrm{~s}$ rRNA served as the internal control.

\section{Western Blot}

Western blot analyses were performed as previously described[17]. The primary antibodies 
included: Angiogenin (Sc-74528, Santa cruz), HMGA2 (ab52039, Abcam) and Lamin B 2 (ab151735, Abcam).

\section{Immunocytochemistry}

In immunohistochemistry, indirect fluorescent staining was performed with Cy2-conjugated donkey anti-rabbit secondary antibody (Jackson Labs, Bar Harbor, ME, USA). Primary antibody is rabbit anti-HMGA2 (ab52039, Abcam).

\section{Chromatin Immunoprecipitation}

The chromatin immunoprecipitation (ChIP) assay was performed using the EZ-ChIP Chromatin Immunoprecipitation Kit (EMD Millipore) under the manufacturer's instructions. Forty-eight hours after infected with Ad-ANG, the SK-MES-1s cells were treated with fresh culture medium containing formaldehyde (formaldehyde final concentration 1\%) for 10 minutes at room temperature on a rocking platform $(80 \mathrm{rpm})$. Then the cells were treated with glycine solution (glycine final concentration $0.125 \mathrm{~mol} / \mathrm{L}$ ) for 5 minutes. After washed by PBS, the cells were collected and lysed. DNA sonication was performed using a VCX400 ultrasonic processor (Sonics \& Materials, Newtown, Conn). The immunoprecipitating antibody of ANG was antiangiogenin antibody (Sc-74528, Santa cruz). For a negative control, normal mouse IgG in the EZ-ChIP Kit was used. The sequences of primers used were as follows: Forward 5'-TGAGTGCAATTGTGGTGTTAG G-3'; Reverse 5'-CTAGAGGCAACCGAAGTTCC-3' (amplification position: -847 to $-947 \mathrm{bp}$ upstream of the transcription start site of HMGA2). For semi-qPCR amplifications were performed with 35 cycles in a total volume of $20 \mu \mathrm{L}$ and run on a $2 \%$ agarose gel. For RT-PCR the difference between the negative control and ANG varying at least 3 cycles was considered significant.

\section{Luciferase assay}

A DNA fragment (HMGA2 WT) of -1 to $-1500 \mathrm{bp}$ of the transcription start site (TSS) of HMGA2 gene was chemically synthesized. The chemical synthesis products were cloned into a psiCHECK-2 basic vector upstream of the luciferase gene. The plasmid psiCHECK-2-HMGA2-WT was constructed using the following primers: forward, 5'-TCACATGGCTCGAC AGATCTTCTCTCTCCATTACAGCTAA-3', and reverse, 5'-TGGAAGCCATGGTGGCTAGCACTCAC ACACACTCATCCCA-3'. The underlined sequences indicate the restriction enzyme sites for Bg1II and NheI, respectively. A DNA fragment (HMGA2 MU) of -1 to $-846 \mathrm{bp}$ and -948 to $-1500 \mathrm{bp}$ (without the ANG binding region obtained from ChIP results) of the TSS of HMGA2 was also chemically synthesized and the plasmid psiCHECK-2-HMGA2-MU was constructed.
The reporter constructs were transfected into SK-MES-1s cells. Luciferase activity was measured 48 hours after transfection, using the Dual-Luciferase Reporter Assay (Promega). The firefly luciferase activity was normalized by renilla luciferase activity to eliminate the influence of any transfection efficiency difference.

\section{Cell migration}

Transwell migration chambers were used to investigate cell migration ability. In brief, Transwell migration assay was performed in a 24-well Transwell chamber (pore size, $8 \mu \mathrm{m}$; Corning). Thirty-six hours after infection, $0.5 \times 10^{5}$ cells were plated into the upper chamber with non-coated membrane. The cells were then incubated for $8 \mathrm{~h}$. Cells that did not migrate through the pores were removed with a cotton swab. Migrated cells were fixed, stained in a $0.1 \%$ crystal violet solution and counted.

\section{Invasion assay}

The invasion assay was performed using Transwell insert chambers with a pore size of $8 \mu \mathrm{m}$ (Corning). The Transwell filter inserts were coated with matrigel; $0.5 \times 10^{5}$ cells were seeded in serum-free medium in the upper chamber. After $24 \mathrm{~h}$ incubation at $37^{\circ} \mathrm{C}$, cells in the upper chamber were carefully removed with a cotton swab and the cells that had traversed the membrane were fixed, stained in a $0.1 \%$ crystal violet solution and counted.

\section{Cell proliferation}

Cell proliferation was analyzed using a Cell Counting Kit-8(Beyotime, China). Twelve hours after plated into a 96-well at a density of 2000 cells/well. Cells were transfected with adenovirus. Cells were incubated for $0,24,48,72 \mathrm{~h} ; 10 \mu \mathrm{L}$ CCK8 solution was added to each well and the cultures were incubated at $37^{\circ} \mathrm{C}$ for $1 \mathrm{~h}$. After that absorbance at $450 \mathrm{~nm}$ was measured.

\section{Cell apoptosis}

Cell apoptosis was analyzed by Annexin V-FITC assay. Briefly, cells were stained with Annexin V-FITC and propidium iodide (PI) using the ANNEXIN V-FITC Kit (Beckman) according to the manufacturer's protocol and subjected to flow cytometric analysis. Viable cells were not stained by Annexin V or PI; early apoptotic cells were stained by Annexin V but not PI whilst late apoptotic cells were stained by Annexin V and PI.

\section{PCR array}

Ad-GFP SK-MES-1s and Ad-ANG SK-MES-1s were used for PCR array analysis. A custom SABiosciences PCR array (QIAGEN) was created 
incorporating 84 genes generated from primary literature that concerning cell proliferation, invasion and apoptosis. Total cellular RNA was converted to cDNA using the RT ${ }^{2}$ First Strand Kit (QIAGEN). Real time PCR was performed running on a LightCycler ${ }^{\circledR}$ 480 Real-Time PCR System (Roche Diagnostics). Five housekeeping genes (GAPDH, $\beta$-actin, 18 s rRNA, TBP and RPLP1) were used for normalizing the expression levels of target genes aforementioned. A threshold value of 3.5 was used to identify genes of interest. Heat map was generated using freeware TM4-MEV software.

\section{Statistical analysis}

All statistical analyses were performed by SPSS version 19.0. The quantitative data was first evaluated whether they followed the normal distribution by the Shapiro-Wilk test. The data of non-normal distribution was performed by Kruskal-Wallis test. The data of normal distribution was performed by Student's $t$ test. $P$-value less than 0.05 was considered statistically significant difference.

\section{Results}

\section{ANG expression is increased in the lung squamous cell carcinoma tissue}

Quantitative RT-PCR and western-blot were used to examine the expression levels of ANG in normal tissue as well as cancerous tissue in SQCLC patients. qRT-PCR results showed that ANG mRNA levels were significantly higher in cancerous tissue, as compared with the normal adjacent tissue (Figure 1, A). Similarly, western-blot results showed a higher local expression levels of ANG protein in cancerous tissue in SQCLC patients (Figure 1, B).

\section{ANG regulates proliferation, invasion and apoptosis of SK-MES-1 cells in vitro}

Since the local expression level of ANG considerably increased in the cancerous tissue of SQCLC patients, as compared with the adjacent normal tissue, we considered that ANG might play an important role in the development of SQCLC. To investigate the effect of ANG in SQCLC, we applied gain- and loss-of function strategies in an in vitro system. Firstly, we infected SK-MES-1 cells with Ad-ANG and the infection efficiency reached $\leq 70 \%$ (Figure 1, C). Cells infected with Ad-GFP were used as control. The expression levels of ANG were examined 48 hours after infection. The relative mRNA level of ANG in the Ad-ANG SK-MES-1 cells was 11.77, as compared with the Ad-GFP cells (Figure 1, D). An increased expression of ANG protein in the Ad-ANG SK-MES-1 was also observed according to western blot results (Figure 1, E).
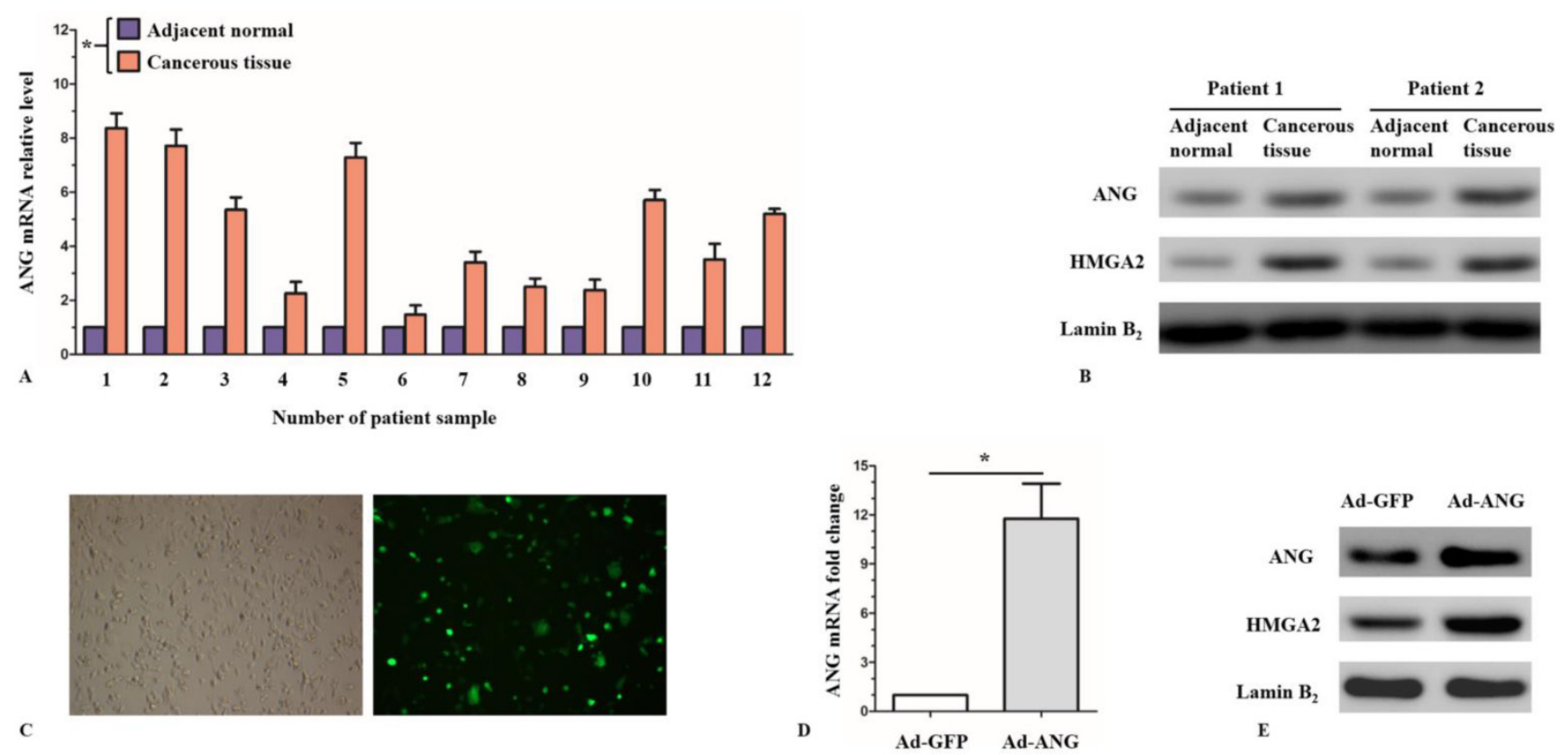

Figure 1. ANG expression levels in the SQCLC cancerous tissues versus adjacent normal tissues, and verification of ANG overexpression in Ad-ANG SK-MES-1s. A, RT-PCR showed that ANG mRNA levels were significantly higher in cancerous tissues than in adjacent normal tissues in SQCLC patients ( $n=3$ experiments for all tests). $* P<0.05$ versus adjacent normal. B, Local expressions of ANG and HMGA2 protein were upregulated in cancerous tissues. C, The transfection efficiency of adenovirus vectors in SK-MES-1s, which was assessed under fluorescence microscope, reached $\leq 70 \%$. D, mRNA level of ANG was significantly enhanced in Ad-ANG SK-MES-1s ( $=3$ for each cell group). $* P$ $<0.05$ versus control. Ad-GFP served as control. E, Protein levels of ANG and HMGA2 were significantly upregulated in Ad-ANG SK-MES-1s. All experiments were carried out in triplicate. 

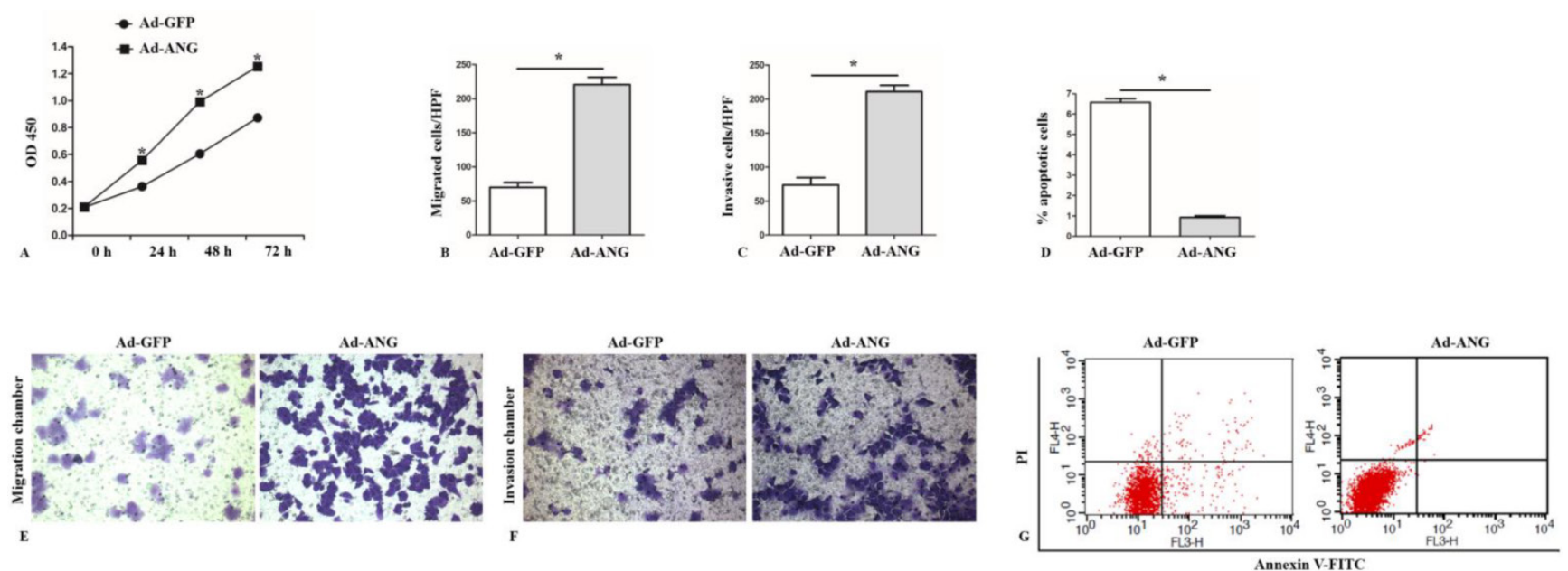

Figure 2. ANG is an important regulator of proliferation, migration, invasion, and apoptosis of SK-MES-1s. A, A higher optical density at 450 nm (OD 450$)$ in the CCK 8 assay was observed in Ad-ANG SK-MES-1s at 24,48 , and 72 hours $(n=3$ for each cell group). $* P<0.05$ versus control. $B$ and $E$, Results of the migration chamber assay showed that migration capability was significantly higher in the Ad-ANG SK-MES-1s. Quantified data were presented as the number of migrated cells per high-power field (HPF) ( $\mathrm{n}=3$ for each cell group). $* P<0.05$ versus control. $C$ and $\mathrm{F}$, Transwell invasion results showed that invasion capability was significantly higher in Ad-ANG SK-MES-1s. Quantified data were presented as the number of invasion cells per HPF ( $n=3$ for each cell group). $* P<0.05$ versus control. $D$ and G, Cells were stained with Annexin V-FITC and propidium iodide (PI). Flow cytometric contour plots showed the percentage of the stained cells. Histogram showed the percentage of Annexin $\mathrm{V}^{+} / \mathrm{PI}-$ and Annexin $\mathrm{V}^{+} / \mathrm{PI}+$ cells. Ad-ANG cells showed a lower apoptotic rate $(\mathrm{n}=3$ each cell group). $* P<0.05$ versus control. All experiments were carried out in triplicate.

Considering the recognition of $\mathrm{ANG}^{\prime}$ s important role in regulating the proliferation, invasion and apoptosis of some critical cells in a series of diseases[8, 18, 19], the regulation of ANG on proliferation, invasion and apoptosis of SK-MES-1 cells was investigated

Cell proliferation examination was performed after infection using a CCK8 assay. It was found that Ad-ANG SK-MES-1s manifested increased proliferation level at 24 hours, 48 hours and 72 hours after infection (Figure 2, A).

With regard to SK-MES-1 cells migration and invasion capability evaluation, transwell migration and invasion chamber assays were applied to assess the impact of ANG on these cell properties. Results showed that Ad-ANG SK-MES-1s presented not only significantly increased migration but also invasion capability (Figure 2, B, C, E, and F), as compared with the Ad-GFP cells.

Using Annexin V-FITC assay, we found that the percentage of Annexin $\mathrm{V}-\mathrm{FITC}^{+} / \mathrm{PI}^{-}$and Annexin V-FITC ${ }^{+} / \mathrm{PI}^{+}$in Ad-ANG cells were both significantly lower than those in Ad-GFP cells (Figure 2, D and G).

Next, SK-MES-1 cells were infected with ANG shRNA (shANG) and cultured for 48 hours. Scrambled shRNA (shScramble) served as the negative control. Compared with shScramble SK-MES-1s, the expression level of ANG mRNA in shANG cells decreased by $73.3 \%$ (Figure 3, A). Western blot results also confirmed lower expression level of ANG in shANG SK-MES-1s (Figure 3, B). As expected, decreased proliferation and migration capacity was observed in shANG cells (Figure 3, C, D and E).
These results definitely indicated that ANG was an important regulator of proliferation, migration, invasion, and apoptosis in SK-MES-1s.

\section{Analysis of PCR array in Ad-ANG and Ad-GFP SK-MES- $1 \mathrm{~s}$ and HMGA2 expression in lung squamous cell carcinoma patients}

To demonstrate the mechanism of ANG's regulation effect on proliferation, migration, invasion, and apoptosis of SK-MES-1 cells, PCR array analysis was utilized to preliminarily figure out the potential target genes of ANG by comparing the relative expression level differences of totally 84 candidate genes concerning cell proliferation, invasion and apoptosis between Ad-ANG SK-MES-1s and Ad-GFP SK-MES-1s (Figure 4, A). Results showed that there were significant up-regulation on mRNA expression levels of MMP-2, HMGA2, NF-kB and BCL-2 in the Ad-ANG group, as compared with the control group. The mRNA expression levels of MCP-1, FHL3 and p65, on the other hand, were down-regulated in Ad-ANG SK-MES-1 cells compared with Ad-GFP cells (Figure 4, B).

The high mobility group A (HMGA2) proteins are small non-histone chromosomal proteins which are characterized by three highly conserved DNA binding motifs called "AT-hooks" and an acidic tail. They preferentially bind to the minor groove of AT-rich B form DNA by recognizing a particular DNA structure rather than a specific nucleotide sequence[20]. Gene regulatory mechanisms seem to be involved in reactivation of HMGA2 expression in malignant tumors. 

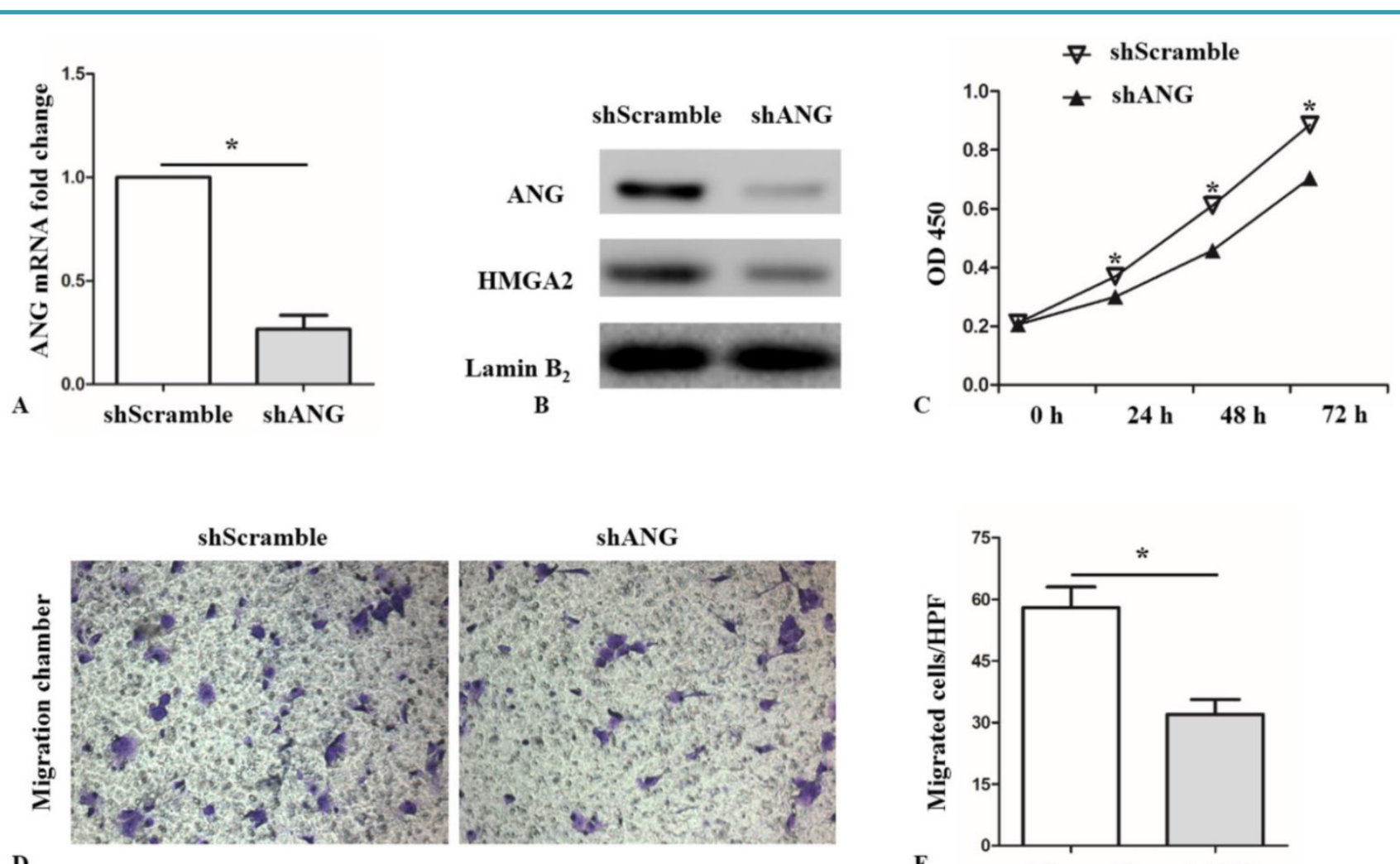

D

Figure 3. Knocking down of ANG suppresses proliferation and migration abilities of SK-MES-1s. A and B, Successful knockdown of ANG in SK-MES-1s via shANG. HMGA2 protein expression was decreased in shANG SK-MES-1s, as compared with shScramble SK-MES- $1 \mathrm{~s}(\mathrm{n}=3$ for each cell group). $* P<0.05$ versus control. C, shANG SK-MES-1s showed a lower proliferation capacity at 24,48 , and 72 hours, as compared with shScramble SK-MES- $1 \mathrm{~s}(\mathrm{n}=3$ for each cell group). $* P<0.05$ versus control. D and E, migration capability was significantly lower in the shANG SK-MES- Is. Quantified data were presented as the number of migrated cells per HPF $(n=3$ for each cell group). *P<0.05 versus control. All experiments were carried out in triplicate.

However, it is still uncertain whether HMGA2 is involved in the pathogeneses of SQCLC. To clarify this issue, we examined the expression levels of HMGA2 in the cancerous tissue and normal adjacent tissue of SQCLC patients. It was found that the HMGA2 mRNA levels was significantly higher in the cancerous tissue (Figure 4, C). The western-blot result further confirmed an increased HMGA2 protein levels in the lung cancerous tissue, as compared with normal adjacent tissue (Figure 1, B). These results, taken together, suggested that HMGA2 might be involved in the pathogeneses of SQCLC.

\section{ANG directly regulates HMGA2 expression in SK-MES-1s}

Although we had got a positive PCR array analysis result aforementioned, it is still uncertain whether ANG would regulate the expression of HMGA2 in SK-MES-1 cells. We thus further confirmed through qRT-PCR and western blot that HMGA2 mRNA and protein levels were both significantly up-regulated in Ad-ANG SK-MES-1 cells, as compared with the Ad-GFP cells (Fiugre 1, E and Figure 4, D). We also found that HMGA2 mRNA and protein levels were both decreased in shANG SK-MES-1 cells (Figure 3, B and Figure 4, E).
Moreover, immunocytochemistry showed HMGA2 expression levels were decreased in the nucleus of shANG SK-MES-1 cells (Figure 4, F). These results implied that ANG would regulate HMGA2 expression in SK-MES-1s.

Taken into account that ANG is a transcription factor and would directly regulate its target gene[8, 19]. These interesting results prompted us the consideration that if HMGA2 was directly regulated by ANG in SK-MES-1 cells. To clarify this hypothesis, we firstly used ChIP assay to examine whether ANG is recruited to the promoter of HMGA2. DNA enrichment was measured by RT-PCR and semi-qPCR, respectively. Results from ChIP assay showed that ANG directly bound to the promoter of HMGA2 (Figure 5, A). Next, we constructed a plasmid psiCHECK-2-HMGA2-WT containing -1 to $-1500 \mathrm{bp}$ of the TSS of HMGA2 (Figure 5, B). The enhancement of HMGA2 promoter activity by overexpression of ANG was verified in SK-MES-1 cells (Figure 5, C). To further confirm that the ANG binding region obtained from ChIP assay led to the aforementioned phenomenon, we constructed a mutant plasmid psiCHECK-2-HMGA2-MU: -1 to -846 bp and -948 to $-1500 \mathrm{bp}$ of the TSS of HMGA2 (Figure $5, \mathrm{~B})$. Overexpression of ANG did not enhance the 
promoter activity of psiCHECK-2-HMGA2-MU (Figure 5, C). These findings, for the first time, demonstrate that HMGA2 is a direct regulation target of ANG during transcription in SK-MES-1 cells.

\section{HMGA2 mediates the effects of ANG on proliferation, migration, and invasion in SK-MES-1s}

Based on the fact that HMGA2 was considerably reported to be involved in cell invasion[21], it is further assessed whether the effects of ANG on proliferation, invasion and apoptosis in SK-MES-1 cells were mediated via HMGA2. To confirm this hypothesis, we infected Ad-ANG SK-MES-1 with shHMGA2. As expected, the HMGA2 mRNA and protein levels in the Ad-ANG/shHMGA2 SK-MES-1 cells were effectively decreased, compared with the Ad-ANG/shScramble cells (Figure 6, A and B). Furthermore, it was found that Ad-ANG/shHMGA2 SK-MES-1 cells showed decreased proliferation capability (Figure 6, C). Meanwhile, decreased migration and invasion capacity was also observed in Ad-ANG/shHMGA2 cells (Figure 6, D-G). However, knocking down of HMGA2 did not enhance the

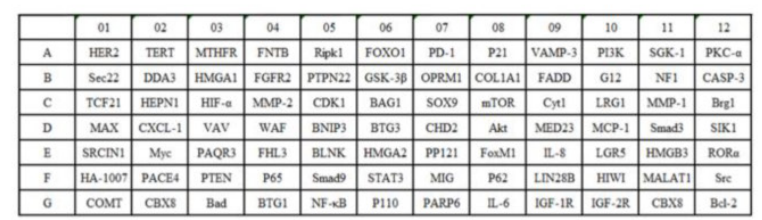

$\begin{array}{lllllllllllll}A & 01 & 02 & 03 & 04 & 05 & 06 & 07 & 08 & 09 & 10 & 11 & 12\end{array}$
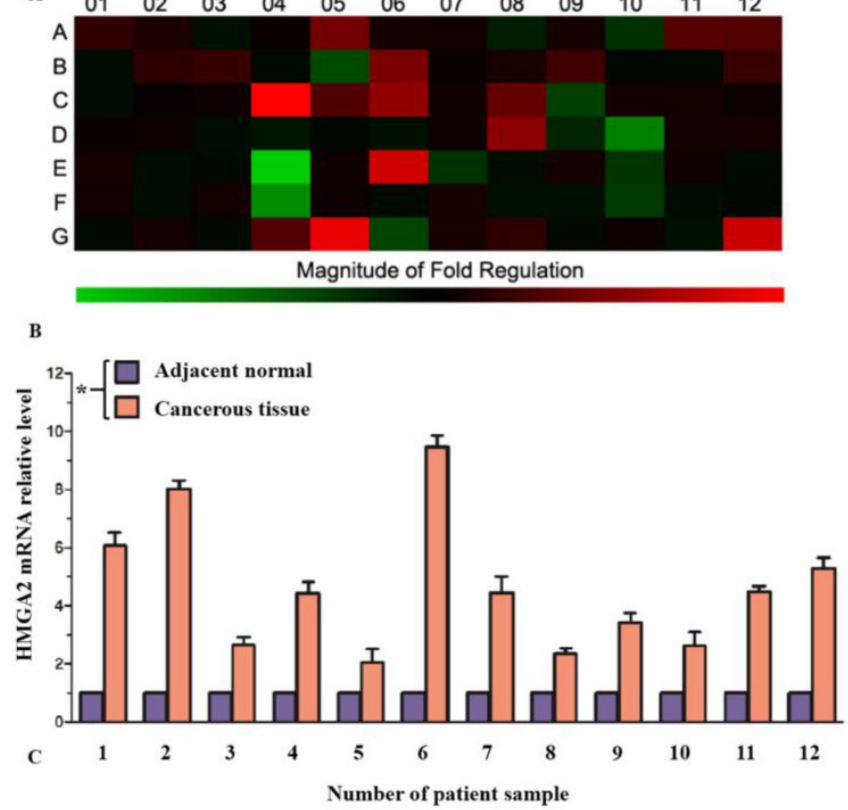

Figure 4. PCR array analysis of candidate ANG target genes, ANG regulates HMGA2 expression in SK-MES-1s. A, Names of the 84 proliferation, migration, and invasion related genes. B, Heat map of the variations in the expression of the 84 genes between Ad-ANG and Ad-GFP SK-MES-1s. C, HMGA2 mRNA levels were significantly higher in cancerous tissues than in adjacent normal tissues in SQCLC patients $(n=3$ experiments for all tests). $*<0.05$ versus adjacent normal. D, HMGA2 mRNA expression was activated in Ad-ANG SK-MES-1s ( $n=3$ for each cell group). $* P<0.05$ versus control. E, HMGA2 mRNA expression was decreased in shANG SK-MES-1s, as compared with shScramble SK-MES-1s ( $\mathrm{n}=3$ for each cell group). $* P<0.05$ versus control. $\mathrm{F}, \mathrm{ICC}$ results showed that HMGA2 expression levels were decreased in the nucleus of shANG SK-MES-1s. All experiments were carried out in triplicate. apoptotic rate in Ad-ANG SK-MES-1 cells (Figure 6, $\mathrm{H})$. These results revealed that the regulation effects of ANG on proliferation, migration, and invasion in SK-MES-1 cells were, at least partially, mediated by HMGA2.

\section{Discussion}

The present study reports two novel findings: (i) patients with lung squamous cell carcinoma have increased ANG and HMGA2 in the tumor and (ii) ANG promotes the invasion and proliferation of SQCLC cells through the mediator HMGA2 in vitro.

Previous studies suggested that ANG, as a basic, single-chain protein, was once thought to promote cancer progression by its angiogenic activity[5]. Latter studies indicated that ANG, taking part in cancer development by stimulating both angiogenesis and cancer cell proliferation, mediates DHT-stimulated rRNA transcription in androgen-sensitive LNCaP cells and ANG overexpression permits LNCaP cell growth[22]. Angiogenin could also interact with ribonuclease inhibitor regulating $\mathrm{PI} 3 \mathrm{~K} / \mathrm{AKT} / \mathrm{mTOR}$ signaling pathway in bladder cancer cells[23].

D
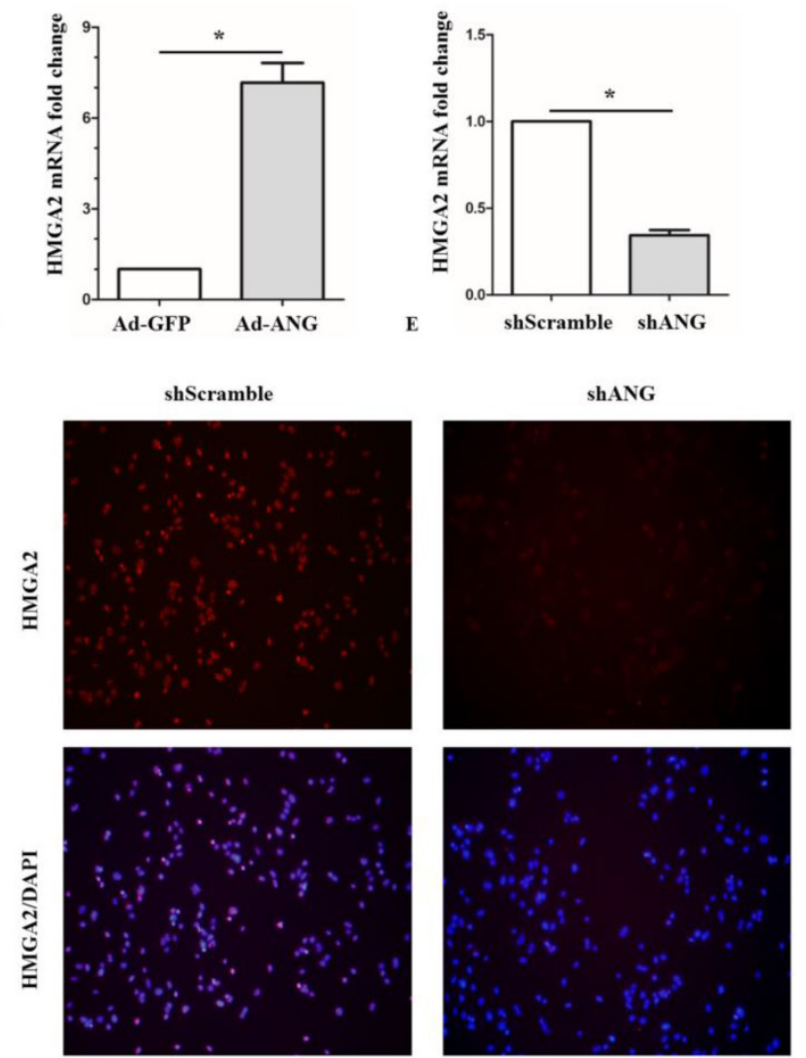

$\mathbf{F}$ 


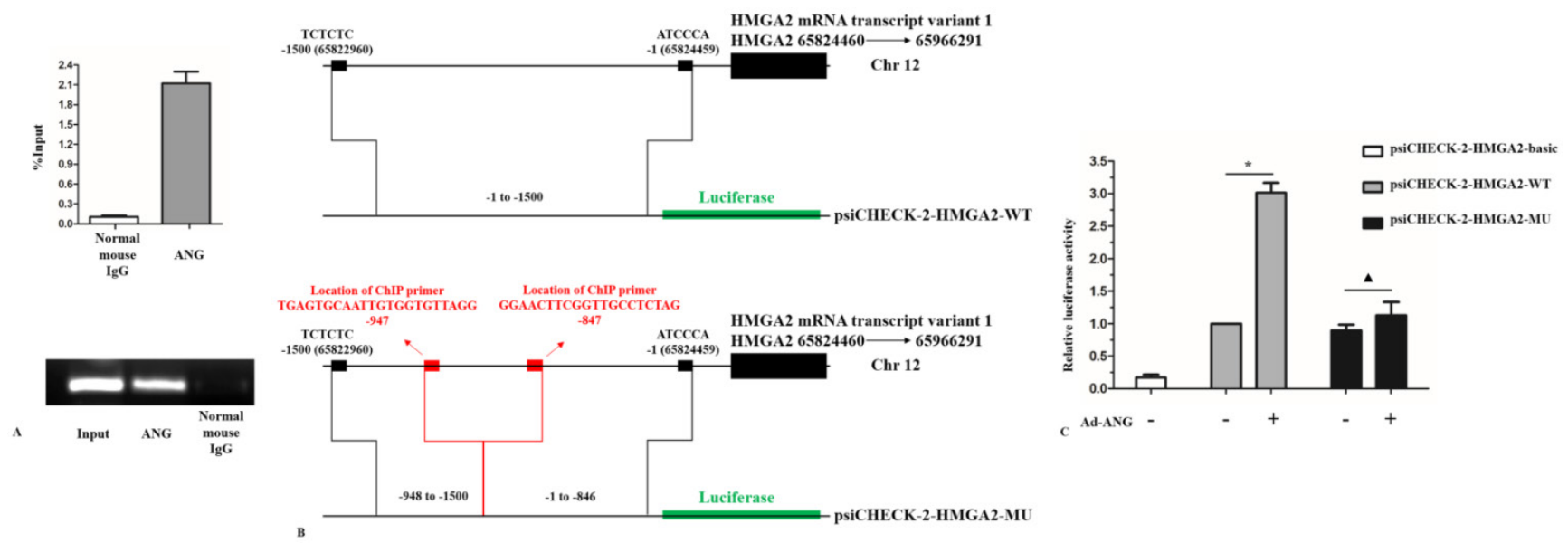

Figure 5. HMGA2 is a direct target of ANG. A, ChIP results showed that ANG was recruited to the promoter of HMGA2 during transcription activity $(\mathrm{n}=3$ experiments for all tests). B, Construction of plasmid psiCHECK-2-HMGA2-WT and plasmid psiCHECK-2-HMGA2-MU. C, Overexpression of ANG significantly enhanced the relative luciferase activity in psiCHECK-2-HMGA2-WT group. However, the relative luciferase activity difference in psiCHECK-2-HMGA2-MU group did not show statistical significance by overexpression of ANG ( $n=3$ experiments for all tests). $* P<0.05$ versus control. ${ }^{\wedge} P>0.05$ versus control. All experiments were carried out in triplicate.
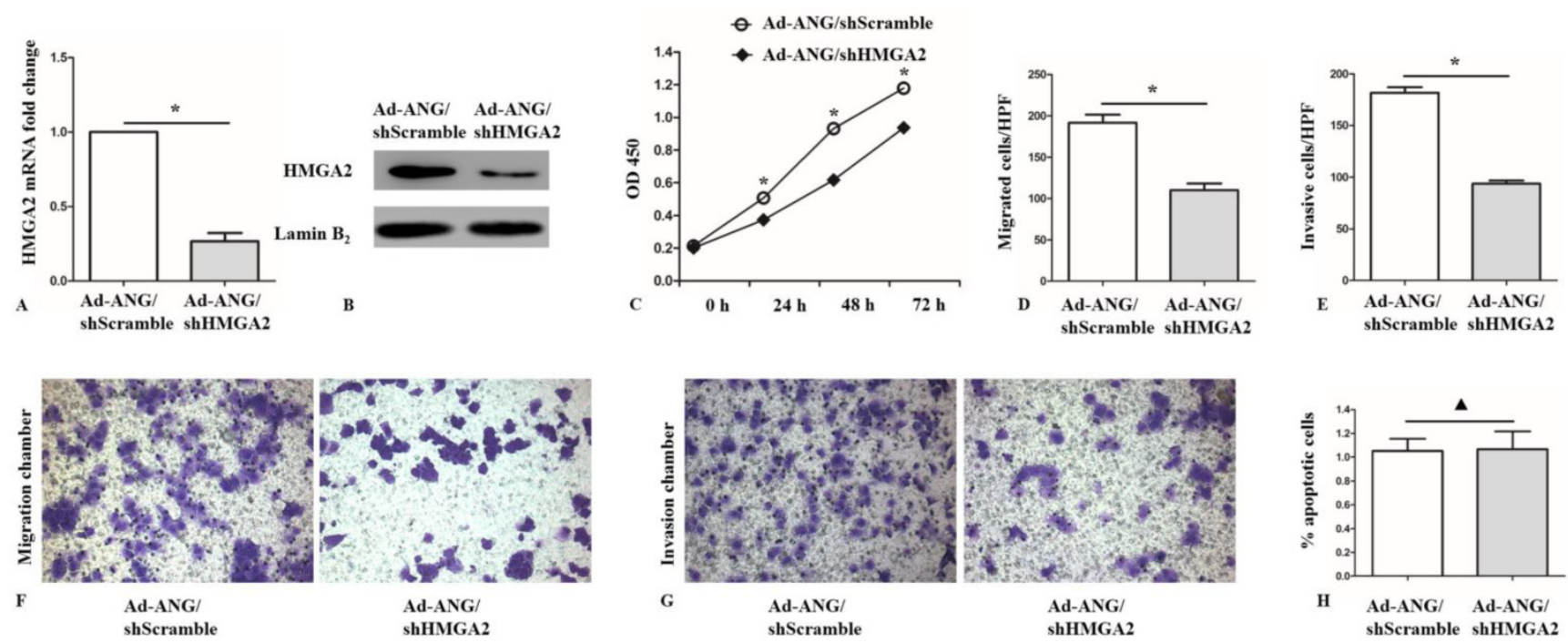

Figure 6. HMGA2 is a mediator of ANG in regulating proliferation, migration, and invasion of SK-MES-1s. A and B, Successful knockdown of HMGA2 in Ad-ANG SK-MES-1s. Ad-ANG SK-MES-1s transfected with shScramble served as negative control ( $n=3$ for each cell group). $* P<0.05$ versus control. C, CCK8 assay after HMGA2 knockdown in Ad-ANG SK-MES-1s. A lower OD 450 value was observed in Ad-ANG/shHMGA2 SK-MES-1s at 24, 48, and 72 hours ( $n=3$ for each cell group). $* P<0.05$ versus control. D and F, Ad-ANG/shHMGA2 cells showed a lower migration ability, as compared to Ad-ANG/shScramble SK-MES-1s. Quantified data were presented as the number of migrated cells per HPF ( $\mathrm{n}=3$ for each cell group). $* P<0.05$ versus control. $E$ and $\mathrm{G}$, Transwell invasion results showed that invasion capability was significantly lower in Ad-ANG/shHMGA2 SK-MES-1s. Quantified data were presented as the number of invasion cells per HPF ( $n=3$ for each cell group). * $P<0.05$ versus control. $H$, The apoptotic rates difference in Ad-ANG/shHMGA2 and Ad-ANG/shScramble SK-MES-1s showed no statistical significance $\left(n=3\right.$ for each cell group). ${ }^{\wedge} P>0.05$ versus control. All experiments were carried out in triplicate.

Our previous study suggested that angiogenin nuclear expression played a dual role in lung adenocarcinoma, with respect to cancer cell proliferation and angiogenesis, and that angiogenin may serve as a potential molecular target for the treatment of human lung AdC[24]. However, so far, the expression pattern of ANG in SQCLC has seldom been studied, with little investigation on the expression of ANG in human tumors. It is reported that the mean level of mRNA ANG expression in MIBC was fourfold higher than in NMIBC and fivefold higher than in benign tissues[10]. Furthermore, Li S et al. found a correlation between increased tissue levels of ANG and cancer patients with aggressive disease and poor prognosis[22]. However, further studies are needed to elucidate the mechanisms of ANG in lung squamous cell carcinoma, which is the very reason that we focused on ANG in the present study. Previously studies have suggested that HMGA2 expression as potential indicator for lung cancer and HMGA2 is highly expressed in metastatic lung adenocarcinoma[25, 26], which showed that HMGA2 promotes lung cancer progression in mouse and human cells by operating as a competing endogenous RNA (ceRNA) for the let-7 microRNA (miRNA) family. Recent studies also 
showed that HMGA2, associated with chromatin throughout the cell cycle in pluripotent human ES cells, whose expression levels are further elevated during human embryoid body formation, seems to be involved in the regulation of key human genes linked to mesenchymal cell lineage differentiation, adipogenesis, and human ES cell proliferation control.[27, 28] Based on these evidences, it's not hard to explain the involvement of HMGA2 in regulation of proliferation and invasion in lung squamous cell carcinoma cells. Herewith, we provided the evidences of HMGA2 and chromatin remodeling in regulation of HMGA2 in lung squamous cell carcinoma cells.

In this study, we examined the expression of ANG in clinical SQCLC tissues by Western blotting and qRT-PCR. The results of qRT-PCR analysis showed that ANG and HMGA2 mRNA level in SQCLC cancerous tissue revealed more increases compared with that in the benign tissues, and the ANG mRNA expression showed a linear correlation of the HMGA2 expression in SQCLC cancerous tissue. It suggested that ANG might play a role in the tumorigenesis of SQCLC.

To extend our clinical studies and investigate its biological function, we employed adenovirus to over-express ANG and siRNA to knockdown ANG expression in SQCLC cell line SK-MES-1, which thus suggested that knockdowning of ANG could become a negative factor for expression of HMGA2. In addition, over-expression of ANG could enhance the ability of proliferation and invasion of lung squamous carcinoma cells in vitro. But when over-expressed ANG along with silencing HMGA2, the ability of cells could weaken.

Collectively, based on these data, it is concluded that angiogenin nuclear expression played an important role in lung squamous carcinoma, as in with respect to cancer cell proliferation and invasion, and that angiogenin may serve as a potential molecular target for the treatment of human lung squamous carcinoma.

\section{Acknowledgements}

This work was supported by the National Natural Science Foundation of China (81272592 and 81301829).

\section{Competing Interests}

The authors have declared that no competing interest exists.

\section{References}

1. Jemal A, Siegel R, Ward E, Murray T, Xu J, Smigal C, et al. Cancer statistics, 2006. CA: a cancer journal for clinicians. 2006; 56: 106-30.

2. Tan SX, Hu RC, Tan YL, Liu JJ, Liu WE. Promoter methylation-mediated downregulation of PRDM5 contributes to the development of lung squamous cell carcinoma. Tumour biology : the journal of the International Society for Oncodevelopmental Biology and Medicine. 2014; 35: 4509-16.

3. Lortet-Tieulent J, Soerjomataram I, Ferlay J, Rutherford M, Weiderpass E, Bray F. International trends in lung cancer incidence by histological subtype: adenocarcinoma stabilizing in men but still increasing in women. Lung cancer (Amsterdam, Netherlands). 2014; 84: 13-22.

4. $\mathrm{Li} \mathrm{S}, \mathrm{Hu}$ GF. Angiogenin-mediated rRNA transcription in cancer and neurodegeneration. International journal of biochemistry and molecular biology. 2010; 1: 26-35.

5. Fett JW, Strydom DJ, Lobb RR, Alderman EM, Bethune JL, Riordan JF, et al. Isolation and characterization of angiogenin, an angiogenic protein from human carcinoma cells. Biochemistry. 1985; 24: 5480-6.

6. Ibaragi S, Yoshioka N, Li S, Hu MG, Hirukawa S, Sadow PM, et al. Neamine inhibits prostate cancer growth by suppressing angiogenin-mediated rRNA transcription. Clinical cancer research : an official journal of the American Association for Cancer Research. 2009; 15: 1981-8.

7. Soncin F, Shapiro R, Fett JW. A cell-surface proteoglycan mediates human adenocarcinoma HT-29 cell adhesion to human angiogenin. The Journal of biological chemistry. 1994; 269: 8999-9005.

8. Tsuji T, Sun Y, Kishimoto K, Olson KA, Liu S, Hirukawa S, et al. Angiogenin is translocated to the nucleus of HeLa cells and is involved in ribosomal RNA transcription and cell proliferation. Cancer research. 2005; 65: 1352-60.

9. Yoshioka N, Wang L, Kishimoto K, Tsuji T, Hu GF. A therapeutic target for prostate cancer based on angiogenin-stimulated angiogenesis and cancer cell proliferation. Proceedings of the National Academy of Sciences of the United States of America. 2006; 103: 14519-24.

10. Miyake H, Hara I, Yamanaka K, Gohji K, Arakawa S, Kamidono S. Increased angiogenin expression in the tumor tissue and serum of urothelial carcinoma patients is related to disease progression and recurrence. Cancer. 1999; 86: 316-24.

11. Mahajan A, Liu Z, Gellert L, Zou X, Yang G, Lee P, et al. HMGA2: a biomarker significantly overexpressed in high-grade ovarian serous carcinoma. Modern pathology : an official journal of the United States and Canadian Academy of Pathology, Inc. 2010; 23: 673-81.

12. Wolffe AP. Architectural transcription factors. Science (New York, NY). 1994; 264: 1100-1.

13. Bustin M, Reeves R. High-mobility-group chromosomal proteins: architectural components that facilitate chromatin function. Progress in nucleic acid research and molecular biology. 1996; 54: 35-100.

14. Langelotz C, Schmid P, Jakob C, Heider U, Wernecke KD, Possinger K, et al. Expression of high-mobility-group-protein HMGI-C mRNA in the peripheral blood is an independent poor prognostic indicator for survival in metastatic breast cancer. British journal of cancer. 2003; 88: 1406-10.

15. Xi YN, Xin XY, Ye HM. Effects of HMGA2 on malignant degree, invasion, metastasis, proliferation and cellular morphology of ovarian cancer cells. Asian Pacific journal of tropical medicine. 2014; 7: 289-92.

16. Wend $\mathrm{P}$, Runke $\mathrm{S}$, Wend $\mathrm{K}$, Anchondo B, Yesayan $\mathrm{M}$, Jardon $\mathrm{M}$, et al. WNT10B/beta-catenin signalling induces HMGA2 and proliferation in metastatic triple-negative breast cancer. EMBO molecular medicine. 2013; 5: 264-79.

17. Wang S, Zhang X, Yuan Y, Tan M, Zhang L, Xue X, et al. BRG1 expression is increased in thoracic aortic aneurysms and regulates proliferation and apoptosis of vascular smooth muscle cells through the long non-coding RNA HIF1A-AS1 in vitro. European journal of cardio-thoracic surgery : official journal of the European Association for Cardio-thoracic Surgery. 2015; 47: 439-46.

18. Jones ML, Ewing CM, Isaacsa WB, Getzenberg RH. Prostate cancer-derived angiogenin stimulates the invasion of prostate fibroblasts. Journal of cellular and molecular medicine. 2012; 16: 193-201.

19. Xia W, Fu W, Cai X, Wang M, Chen H, Xing W, et al. Angiogenin promotes U87MG cell proliferation by activating NF-kappaB signaling pathway and downregulating its binding partner FHL3. PloS one. 2015; 10: e0116983.

20. Reeves R, Nissen MS. The A.T-DNA-binding domain of mammalian high mobility group I chromosomal proteins. A novel peptide motif for recognizing DNA structure. The Journal of biological chemistry. 1990; 265: 8573-82

21. Xia YY, Yin L, Jiang N, Guo WJ, Tian H, Jiang XS, et al. Downregulating HMGA2 attenuates epithelial-mesenchymal transition-induced invasion and migration in nasopharyngeal cancer cells. Biochemical and biophysical research communications. 2015; 463: 357-63.

22. Li S, Hu MG, Sun Y, Yoshioka N, Ibaragi S, Sheng J, et al. Angiogenin mediates androgen-stimulated prostate cancer growth and enables castration resistance. Molecular cancer research : MCR. 2013; 11: 1203-14.

23. Peng Y, Li L, Huang M, Duan C, Zhang L, Chen J. Angiogenin interacts with ribonuclease inhibitor regulating PI3K/AKT/mTOR signaling pathway in bladder cancer cells. Cellular signalling. 2014; 26: 2782-92.

24. Yuan Y, Wang F, Liu XH, Gong DJ, Cheng HZ, Huang SD. Angiogenin is involved in lung adenocarcinoma cell proliferation and angiogenesis. Lung cancer (Amsterdam, Netherlands). 2009; 66: 28-36.

25. Rogalla P, Drechsler K, Schroder-Babo W, Eberhardt K, Bullerdiek J. HMGIC expression patterns in non-small lung cancer and surrounding tissue. Anticancer research. 1998; 18: 3327-30.

26. Kumar MS, Armenteros-Monterroso E, East P, Chakravorty P, Matthews N, Winslow MM, et al. HMGA2 functions as a competing endogenous RNA to promote lung cancer progression. Nature. 2014; 505: 212-7. 
27. Li O, Li J, Droge P. DNA architectural factor and proto-oncogene HMGA2 regulates key developmental genes in pluripotent human embryonic stem cells. FEBS letters. 2007; 581: 3533-7.

28. Li O, Vasudevan D, Davey CA, Droge P. High-level expression of DNA architectural factor HMGA2 and its association with nucleosomes in human embryonic stem cells. Genesis (New York, NY : 2000). 2006; 44: 523-9. 